\title{
Relative Orientation of Pairs of Spiral Galaxies in the Sloan Digital Sky Survey
}

\author{
Jesse Buxton ${ }^{1} \&$ Barbara S. Ryden ${ }^{2}$ \\ Department of Astronomy, The Ohio State University, Columbus, OH 43210 \\ buxton.45@osu.edu, ryden@astronomy.ohio-state.edu
}

\begin{abstract}
We find, from our study of binary spiral galaxies in the Sloan Digital Sky Survey Data Release 6, that the relative orientation of disks in binary spiral galaxies is consistent with their being drawn from a random distribution of orientations. For 747 isolated pairs of luminous disk galaxies, the distribution of $\phi$, the angle between the major axes of the galaxy images, is consistent with a uniform distribution on the interval $\left[0^{\circ}, 90^{\circ}\right]$. With the assumption that the disk galaxies are oblate spheroids, we can compute $\cos \beta$, where $\beta$ is the angle between the rotation axes of the disks. In the case that one galaxy in the binary is face-on or edge-on, the tilt ambiguity is resolved, and $\cos \beta$ can be computed unambiguously. For 94 isolated pairs with at least one face-on member, and for 171 isolated pairs with at least one edge-on member, the distribution of $\cos \beta$ is statistically consistent with the distribution of $\cos i$ for isolated disk galaxies. This result is consistent with random orientations of the disks within pairs.
\end{abstract}

Subject headings: galaxies: fundamental parameters — galaxies: photometry — galaxies: statistics

\section{INTRODUCTION}

The structure of a spiral galaxy is closely tied to its angular momentum distribution. Thus, understanding galaxy formation requires knowledge of how galaxies acquire their angular momentum. The tidal torque model for the acquisition of angular momentum by

\footnotetext{
${ }^{1}$ Department of Physics, The Ohio State University, Columbus, OH 43210

${ }^{2}$ Center for Cosmology \& Astro-Particle Physics, The Ohio State University, Columbus, OH 43210
} 
galaxies (Hoyle 1949; Peebles 1969) states that protogalaxies acquire their angular momentum through the tidal interaction between the protogalaxy and the surrounding nonuniform matter distribution. Spiral galaxies are particularly useful for testing how galaxies acquire angular momentum. Spiral galaxies contain relatively cold, thin disks of stars, gas, and dust, supported primarily by rotation rather than velocity dispersion. These thin disks are unlikely to have suffered a major merger (or large numbers of minor mergers) since their formation; thus, the orientation of a disk's spin angular momentum is unlikely to have changed drastically since the material that formed it was initially torqued.

Binary spiral galaxies are particularly useful test cases for the acquisition of angular momentum. A "binary spiral galaxy" can be defined as a pair of spiral galaxies that are relatively close to each other, but are much farther away from other galaxies of comparable or greater mass. Our galaxy and M31, using this general definition, can be thought of as a binary spiral galaxy. They are separated by $\sim 0.8 \mathrm{Mpc}$, and M33, the next brightest galaxy in the Local Group, is $\sim 2$ magnitudes fainter than our galaxy or M31. If a pair of spiral galaxies is a totally isolated system, with zero net angular momentum initially, then conservation of angular momentum states that the net angular momentum remains zero after the two galaxies have spun each other up. If the net angular momentum of the binary system is, in fact, zero, then the relative orientation of the internal angular momentum vectors of the two galaxies can range from anti-parallel, if the two galaxies are on radial orbits relative to each other, to parallel, if the orbital angular momentum is large.

In the real universe, binary spiral galaxies are never completely isolated. Gott \& Thuan (1978), for instance, in their study of the angular momentum of the Local Group, conclude that most of the angular momentum of our galaxy was produced by M31, but that the tidal torquing by nearby groups, such as the Maffei group $(d \sim 3 \mathrm{Mpc})$ and M81 group $(d \approx 3.6 \mathrm{Mpc})$, was not negligible. Thus, we might expect the distribution of the angle $\alpha$ between the internal angular momentum vectors of the two galaxies in a pair to depend on the number of massive galaxies within several megaparsecs of the pair. In addition, the distribution of $\alpha$ will also depend on the evolutionary history of binary spiral galaxies since they initially attained their angular momentum by tidal torquing. For instance, the merger timescale for a bound pair of galaxies depends on the ellipticity $\varepsilon$ of their mutual orbit (Lacey \& Cole 1993; Jiang et al. 2008), with more nearly radial orbits having a shorter merging timescale. Thus, binary spiral galaxies with radial orbits will more rapidly merge to form an elliptical galaxy. In the case that the binary spiral galaxies are initially isolated, this will result in preferential destruction of the binaries whose internal angular momenta are anti-parallel.

Computer simulations have provided additional insight into the alignment of galaxy 
spins. In $n$-body $\Lambda$ cold dark matter simulations, for instance, massive dark halos $(M \gtrsim$ $3 \times 10^{12} M_{\odot}$ ) have spins that are preferentially oriented in a direction perpendicular to the mass distribution (Paz et al. 2008), and have projected major axes that are aligned with the surrounding galaxy distribution (Paz et al. 2011); these alignments persist on scales up to $\sim 30 \mathrm{Mpc}$. It should be kept in mind, though, that a dark halo is not necessarily a simple structure; Schneider, et al. (2011) show that in the Millennium-2 n-body simulation $\sim 25 \%$ of all halos have nearly perpendicular major axes at large and small radii. Hahn et al. (2010), in a hydrodynamic adaptive mesh refinement simulation that permits disks to form by dissipation, find that at $z=0$, the spin of stellar and gaseous disks are well aligned with the spin of the inner dark halo; however, the alignment between disk spin and the spin of the entire host halo is significantly weaker.

Many studies have been done of the relative orientation of the spin vectors of neighboring spiral galaxies. Three different methods, using increasing amounts of information for each binary spiral galaxy, may be distinguished.

The first method for studying the relative orientation of disk galaxies uses only the position angle of the apparent major axis of each galaxy. If a galaxy is approximated as a rotationally flattened oblate spheroid, then its apparent major axis is at right angles to the projection of its spin axis onto the plane of the sky. The angle $\phi$ between the apparent major axes of the two galaxies in a binary spiral galaxy, defined so that $0^{\circ} \leq \phi \leq 90^{\circ}$, is thus the angle between the projections of the spin axes. If the spin axes of the two spiral galaxies tend to be parallel (implying either parallel or anti-parallel spin vectors), then the distribution of $\phi$ will be weighted toward $\phi=0^{\circ}$; if the spin axes tend to be perpendicular, then the distribution of $\phi$ will be weighted toward $\phi=90^{\circ}$ (Sofue 1992). Employing this method, Sharp et al. (1979), with a sample of 57 pairs of spirals, and Cervantes-Sodi et al. (2010), with 218 pairs of spirals, found distributions for $\phi$ that were statistically consistent with being uniform from $0^{\circ}$ to $90^{\circ}$; these results are consistent with, but do not demand, uncorrelated spin vectors. By contrast, Sofue (1992), using a sample of 390 pairs of spirals, found a significant excess of pairs with $\phi \sim 90^{\circ}$, indicating a tendency for spin axes to be perpendicular.

The second method for studying the relative orientation of spiral galaxies uses the apparent axis ratio $q$ of each galaxy image in addition to the angle $\phi$ between their apparent major axes. If each galaxy is approximated as an oblate spheroid of intrinsic short-to-long axis ratio $\gamma$, then the inclination $i$ of a galaxy with apparent axis ratio $q$ is given by the usual relation

$$
\cos i=\left(\frac{q^{2}-\gamma^{2}}{1-\gamma^{2}}\right)^{1 / 2} \text {. }
$$


Now consider a pair of disk galaxies, with inclinations $i_{1}$ and $i_{2}$, that have an angle $\phi$ between their apparent major axes. If the angular separation of the galaxies is small, then the angle $\beta$ between their spin axes, defined so that $0 \leq \beta \leq 90^{\circ}$, is given by the relation

$$
\cos \beta=\left|\sin i_{1} \sin i_{2} \cos \phi \pm \cos i_{1} \cos i_{2}\right|
$$

The two-fold ambiguity in $\cos \beta$ is due to the tilt ambiguity of the individual galaxies. (If the apparent major axis of a disk galaxy lies in the east-west direction, for example, we don't generally know whether the northern or the southern half of the disk is closer to us.) Using this method, Flin (1993), with a sample of 586 pairs of galaxies, found that their spin axes tend to be parallel. However, this method is intrinsically unable to distinguish between parallel or anti-parallel spin vectors.

The third method for studying relative orientation of spiral galaxies uses additional information to resolve the tilt ambiguity and spin ambiguity of disk galaxies. The tilt ambiguity ("Is the northern or southern half of the disk closer to us?") can be resolved by looking at high-resolution images of a disk galaxy and assuming that dust lanes lie on the outer edge of spiral arms. The spin ambiguity ("Is the disk rotating clockwise or counterclockwise from our point of view?") can be resolved, if the galaxy is close to face-on, by looking at the chirality of the disk's spiral arms and assuming that spiral arms are trailing. Either of these two pieces of information - location of dust lanes and chirality of spiral arms - can be replaced by spectroscopic information about which half of the disk is redshifted relative to the galaxy's nucleus and which is blueshifted. The additional information lets us find the angle $\alpha$ between the spin vectors of the galaxies, defined so that $0 \leq \alpha \leq 180^{\circ}$. Using this method, Helou (1984), with a sample of 31 pairs of galaxies, found that spin vectors tend to be anti-parallel $\left(\alpha \sim 180^{\circ}\right)$. Oosterloo (1993), using a sample of 40 pairs, found that their spin vectors were uncorrelated. Pestaña \& Cabrera (2004) used a sample of 46 pairs of galaxies, largely drawn from the study of Oosterloo (1993), but discarded the information that resolved the spin ambiguity; they then found a tendency for spin axes to be either parallel or perpendicular, with a shortage of pairs at $\beta \sim 45^{\circ}$.

All three methods outlined above for determining relative orientations have shortcomings. Determining the angle $\alpha$ between the spin vectors of two spiral galaxies requires highresolution imaging or spatially resolved spectroscopy. In practice, this has limited sample size; Helou (1984), Oosterloo (1993), and Pestaña \& Cabrera (2004) all had $N<50$ pairs in their samples. In addition, the assumptions must be made that dust lanes are on the outside of spiral arms and that spiral arms are trailing; these assumptions are not invariably true. Determining the angle $\beta$ between the spin axes requires knowing only the apparent axis ratio $q$ for each disk and the angle $\phi$ between their major axes; this can be found from lower resolution images, and permits larger sample sizes. However, because of the tilt ambiguity 
of each disk image, the distribution of $\beta$ is not, in the general case, determined uniquely. In addition, the assumptions must be made that disks are axisymmetric and that their intrinsic short-to-long axis ratio is known; these assumptions are not perfectly true. Determining the distribution of $\phi$, the angle between the apparent major axes of binary spiral galaxies, can be done for even low-resolution images; smearing by the point spread function (PSF) will increase $q$ for a galaxy, but will not strongly affect the position angle unless the PSF is severely asymmetric. This permits large sample sizes. However, even a perfect knowledge of the distribution $F(\phi)$ does not yield a unique solution for $f(\alpha)$, the underlying distribution of angles between the spin vectors.

The problem of determining the relative orientation of disks in binary spiral galaxies is a difficult one. Different investigators, even those using similar techniques or overlapping data sets, find very different conclusions. These range from random orientation of spin axes (Sharp et al. 1979; Oosterloo 1993; Cervantes-Sodi et al. 2010), to a tendency for axes to be parallel (Helou 1984; Flin 1993), to a tendency for axes to be perpendicular (Sofue 1992), to

a tendency for axes to be either parallel or perpendicular (Pestaña \& Cabrera 2004). Our plunge into these troubled waters begins with selecting a sample of close pairs of disk galaxies from the Sloan Digital Sky Survey, as described in Section 2, Our initial analysis, described in Section 3 looks at the distribution of $\phi$, the angle between apparent major axes, for a relatively large $(N=747)$ sample of isolated pairs of galaxies. Then, in Section 4 , we add information about the apparent axis ratios $q$ to find the distribution of $\beta$, the angle between rotation axes. To eliminate the ambiguity in equation (2), we look only at the $N=251$ isolated pairs for which at least one of the galaxies is nearly face-on $(q>0.9)$ or nearly edge-on $(q \leq 0.3)$. Finally, in section 5 , we discuss the implication of our results for the acquisition and evolution of angular momentum in binary spiral galaxies.

\section{DATA SELECTION}

We draw our sample of galaxies from the Sloan Digital Sky Survey Data Release 6 (SDSS DR6), which includes 9853 square degrees of photometric coverage and 7425 square degrees of spectroscopic coverage (Adelman-McCarthy et al. 2008). The photometric imaging is done in 5 bands (ugriz); in this paper, we will use the imaging data in the $r$ band. To build our sample, we start by selecting primary photometric objects that are classified as "galaxies" (obj_type $=3$ ) in the $r$ band and that have spectroscopic data with spectroscopic confidence parameter $\mathrm{zConf}>0.35$. We require that the redshift of each galaxy lie in the range $0.004<z<0.1$; the lower limit on $z$ eliminates contaminating foreground objects, and the upper limit reduces the possibility of weak lensing distortions of apparent shapes, and 
eliminates in practice the necessity of applying K-corrections. To ensure that the galaxies in this sample are well resolved, we include only those images with $\tau>6.25 \tau_{\text {psf }}$, where $\tau$ is the adaptive second-order moment of the galaxy image and $\tau_{\mathrm{psf}}$ is the adaptive secondorder moment of the PSF at the galaxy's location. The sample of galaxies satisfying these constraints contains $N=166,853$ individual galaxies.

To extract a subsample of rotationally supported disk galaxies from our sample of 166,853 galaxies, we start by using the SDSS parameter "fracDeV", which provides a measure of the central concentration of a galaxy's light profile. For each galaxy image, two models are fitted. One model has a de Vaucouleurs profile (de Vaucouleurs 1948):

$$
I(R)=I_{e} \exp \left(-7.67\left[\left(R / R_{e}\right)^{1 / 4}-1\right]\right)
$$

truncated beyond $7 R_{e}$ to go smoothly to zero at $8 R_{e}$. The other model has an exponential profile:

$$
I(R)=I_{e} \exp \left(-1.68\left[R / R_{e}-1\right]\right),
$$

truncated beyond $3 R_{e}$ to go smoothly to zero at $4 R_{e}$. The SDSS data pipeline then takes the best-fitting de Vaucouleurs model and the best-fitting exponential model, and finds the linear combination of the two that best fits the galaxy image. The fraction of the total flux contributed by the de Vaucouleurs component is the parameter fracDeV, which is constrained to lie in the interval $0 \leq$ fracDeV $\leq 1$.

Previous studies using SDSS galaxies have used different cuts in fracDeV to separate late-type (low fracDeV) galaxies from early-type (high fracDeV) galaxies. Chang et al. (2006) and Shao et al. (2007), in their studies of late-type galaxies, found it useful to choose a sample with fracDeV $<0.5$. In this paper, however, our aim is to produce a sample of strongly disk-dominated spiral galaxies. To this end, we make a more stringent cut in the fracDeV parameter, requiring fracDeV $\leq 0.1$. Unterborn \& Ryden (2008) demonstrated that relatively luminous $\left(M_{r} \lesssim-19\right)$ galaxies with fracDeV $<0.1$ have the properties expected of dusty, disk-dominated spiral galaxies: they lie primarily in the blue cloud of the color-magnitude diagram and they have colors and fluxes that are dependent on the apparent axis ratio $q$, with smaller values of $q$ corresponding to redder, fainter galaxies. Moreover, visual inspection of SDSS "postage stamp" images reveals that galaxies with $M_{r} \leq-19$ and fracDeV $<0.1$ appear to be primarily of Hubble type Sbc or Sc (Unterborn \& Ryden 2008).

The inclination-dependent dimming found by Unterborn \& Ryden (2008) in their sample of fracDeV $<0.1$ galaxies is well fitted by the relation $\Delta M_{r}=1.27(\log q)^{2}$. Because the dimming by dust is inclination dependent, a simple flux-limited survey, such as the SDSS spectroscopic survey, will be biased against disks that are nearly edge-on (low q). To provide a correction for this inclination-related bias, we require that all galaxies in our final 
sample would be above the flux limit if they were seen edge-on. If an SDSS galaxy with fracDeV $<0.1$ has $q \leq 0.2$, we assume that it is already edge-on. If it has $q>0.2$, we compute its edge-on flux to be

$$
m_{r}(\operatorname{edge}-\text { on })=m_{r}(\text { obs })+1.27\left[(\log 0.2)^{2}-(\log q)^{2}\right] .
$$

To enter our corrected flux-limited sample, a galaxy must have $m_{r}$ (edge - on $) \leq 17.77$, corresponding to the completeness limit for the SDSS spectroscopic survey for galaxies.

Finally, although disk-dominated spirals are well fitted by exponential profiles, so are other subclasses of galaxies, such as dwarf ellipticals. Unterborn \& Ryden (2008) found that fracDeV $<0.1$ galaxies dimmer than $M_{r} \sim-19$ have a $u-r$ color that is independent of $q$. The lack of inclination-dependent reddening for the relatively low-luminosity galaxies reflects the fact that they are blue $(u-r \sim 1.5)$ dwarf galaxies in which the stars and dust are not in orderly thin disks. To eliminate the dwarf galaxies from our sample, we start by following Unterborn \& Ryden (2008) in computing an approximate face-on absolute magnitude for each galaxy:

$$
M_{r}(\text { face }- \text { on })=M_{r}-1.27(\log q)^{2} .
$$

To exclude the dwarfs, we require that $M_{r}($ face - on $) \leq-19.4$. Throughout this paper, when computing distances and absolute magnitudes, we assume a consensus cosmology with $H_{0}=70 \mathrm{~km} \mathrm{~s}^{-1} \mathrm{Mpc}^{-1}, \Omega_{m, 0}=0.3$ and $\Omega_{\Lambda, 0}=0.7$.

Applying the additional criteria that fracDeV $\leq 0.1, m_{r}$ (edge - on $) \leq 17.77$, and $M_{r}($ face - on $) \leq-19.4$, we reduce our sample size from $N=166,853$ galaxies to $N=32,358$ luminous, disk-dominated spiral galaxies. To select binary spiral galaxies from this sample, we require that the redshift difference between two disk galaxies corresponds to a radial velocity difference $\Delta v \leq 300 \mathrm{~km} \mathrm{~s}^{-1}$, and that the projected separation between them be $R_{p} \leq 1 \mathrm{Mpc}$. To ensure that the binary system is relatively isolated, we also require that no other galaxy in the SDSS DR6 spectroscopic survey be within $\Delta v=300 \mathrm{~km} \mathrm{~s}^{-1}$ and $R_{p}=1 \mathrm{Mpc}$ of either spiral galaxy in the binary system. These criteria leave us with $N_{p}=747$ isolated binary spiral galaxies. If we had chosen $\Delta v=600 \mathrm{~km} \mathrm{~s}^{-1}$ as our critical radial velocity difference (both for defining binaries and for defining isolated binaries), then we would have ended with $N_{p}=699$ isolated binary spiral systems, and the qualitative results of our study would have been unchanged.

As a control, it will be useful to have a sample of luminous disk galaxies that are not part of a binary spiral system, and that are relatively isolated from bright galaxies of all types. To select a sample of isolated disks from our sample of $N=32,358$ luminous, diskdominated spiral galaxies, we require that no other galaxy in the SDSS DR6 spectroscopic survey be within $\Delta v=300 \mathrm{~km} \mathrm{~s}^{-1}$ and $R_{p}=1 \mathrm{Mpc}$ of the spiral galaxy in question. These 
criteria leave us with $N_{i}=16,814$ isolated spiral galaxies. However, it is known that Sloan galaxies in close pairs have a significantly different distribution in redshift and stellar mass than non-paired Sloan galaxies (Ellison et al. 2008; Perez et al. 2009). Thus, to ensure that the control galaxies are similar in their properties to the galaxies in pairs, we adapt the method of Patton et al. (2011) for finding a matched control sample. We first go down the list of $2 N_{p}=1494$ disk galaxies in pairs, and find the isolated galaxy which best matches the redshift of each paired galaxy; once an isolated galaxy is matched to a paired galaxy, it is removed from further consideration. This gives a sample of 1494 isolated galaxies that are very closely matched in redshift to the galaxies in pairs. To increase the number of galaxies in our matched control sample, we iterate this procedure until the redshift distribution of the matched control sample deviates significantly from that of the galaxies in pairs. We found that 7 iterations gave a KS probability of $P_{\mathrm{KS}}=0.998$ for comparison of the redshift distributions; going to an 8th iteration dropped the probability to $P_{\mathrm{KS}} \approx 0.23$. Thus, we adopt a matched control sample with $N_{c}=7 \times 1494=10,458$ galaxies.

Figure 1 shows the cumulative distribution of redshifts for the disk galaxies in pairs (green line), for the matched control sample (red line), and for the complete unmatched sample of 16,814 isolated galaxies (black line). In this plot, the redshift distributions for the disks in pairs and the matched control sample are nearly indistinguishable (and in fact yield a KS probability of nearly one). Note that although we selected our matched control sample on the basis of redshift, and not on both redshift and estimated stellar mass, as Patton et al. (2011) did, we find that selecting purely on redshift gives similar (but not identical) distributions in color and absolute magnitude.

The left panel of Figure 2 shows the cumulative distribution of $g-r$ color for the disks in pairs (green line), for the matched control sample of isolated disks (red line), and the complete sample of isolated disks (black line). The control sample consists of galaxies that are redder than the disks in pairs, at a statistically significant level $\left(P_{\mathrm{KS}}=0.014\right)$; the median color of the control sample is $g-r=0.580$, while the median color of the disks in pairs is $g-r=0.570$. This difference in color may be due in part to starburst triggered in close encounters; Patton et al. (2011) found that "blue cloud" galaxies in close pairs $\left(R_{p}<80 \mathrm{kpc}, \Delta v<200 \mathrm{~km} \mathrm{~s}^{-1}\right.$ ) have $g-r$ colors that are $\sim 0.02$ magnitudes bluer than a sample of galaxies, matched to have the same distribution of redshifts and stellar masses, that are not in close pairs. Since our sample of pairs contains primarily wider pairs (out to $R_{p}=1 \mathrm{Mpc}$ ), it is unsurprising that our color difference, of $\sim 0.01$ magnitudes, is smaller than that found by Patton et al. (2011).

The right panel of Figure 2 shows the cumulative distribution of the corrected (face-on) absolute magnitude $M_{r}^{f}$ for the disks in pairs (green line), the matched control sample of 
isolated disks (red line), and the complete sample of isolated disks (black line). Although the complete sample of isolated galaxies is 0.148 magnitudes brighter, in the median, than the sample of paired galaxies, the matched control sample is 0.026 magnitudes fainter. For the remainder of this paper, we will compare the sample of $N_{p}=747$ disk galaxies in pairs to the matched control sample of isolated galaxies, and not to the complete sample of 16,814 isolated galaxies, which skew toward higher luminosity, redder color, and higher redshift.

The difference in median color between isolated disk galaxies and disk galaxies in pairs cannot be attributed to a difference in inclination-dependent reddening between the two populations. The green line in Figure 3 shows the cumulative distribution of the isophotal axis ratio $q$ for the 1494 disk galaxies in pairs. The red line shows the distribution of $q$ for the matched control sample of isolated galaxies. (If spiral galaxies were randomly oriented, infinitesimally thin, perfectly circular disks, then the distribution would be a straight diagonal line from lower left to upper right on the plot; the lack of galaxies at small $q$ indicates they are not infinitesimally thin and the lack of galaxies at large $q$ indicates they are not perfectly circular.) A Kolmogorov-Smirnov test comparing the shapes of isolated spirals in the matched control sample (red) and the spirals in binaries (green) yields a probability score $P_{\mathrm{KS}}=0.367$, consistent with their being drawn from the same parent distribution.

\section{ANALYSIS: APPARENT MAJOR AXIS ORIENTATION}

The first portion of our analysis involves studying the angle $\phi$ between the projected major axes of the two galaxy images in each of our $N_{p}=747$ isolated binary spiral systems. The angle $\phi$ is given by the relation

$$
\cos \phi=\left|\cos \left(\theta_{1}-\theta_{2}\right)\right|
$$

where $\theta_{1}$ and $\theta_{2}$ are the isophotal position angle for each galaxy in the pair, taken from the $r$ band $25 \mathrm{mag} \mathrm{arcsec}^{-2}$ isophote. If the disks of the two galaxies are randomly oriented with respect to each other in three dimensions, then the distribution of $\phi$ will be uniform in the

interval $\left[0^{\circ}, 90^{\circ}\right.$ (Oosterloo 1993). The cumulative probability distribution for $\phi$ is shown as the black line in Figure 4, the red line is the result for a distribution uniform in the interval $\left[0^{\circ}, 90^{\circ}\right]$. A Kolmogorov-Smirnov test comparing the two distributions yields a probability score $P_{\mathrm{KS}}=0.892$. Thus, the distribution of $\phi$ is consistent with the hypothesis of random disk orientations.

A plot of the angle $\phi$ versus the projected separation $R_{p}$ of the galaxies in each isolated binary is presented in Figure 5. The distribution of points reveals no trends with increasing $R_{p}$; in particular, close pairs have no tendency to be more strongly aligned. A KS test 
comparing the distribution of $\phi$ for the $N=335$ pairs with $R_{p}<0.5 \mathrm{Mpc}$ to the distribution for the $N=412$ pairs with $R_{p} \geq 0.5 \mathrm{Mpc}$ yields $P_{\mathrm{KS}}=0.885$, confirming the visual impression of Figure 5 ,

\section{ANALYSIS: ROTATION AXIS ORIENTATION}

Although a uniform distribution of $\phi$ is consistent with random orientations in three dimensions, it doesn't demand random orientations. In the general case, our knowledge of the distribution of $\beta$, the angle between the spin axes of the galaxies, is impeded by the ambiguity of equation (2). However, if we chose only those pairs which have at least one nearly face-on member, then we can assume an inclination $\cos i_{1} \approx 1$ for one member, and thus

$$
\cos \beta \approx \cos i_{2} \approx\left(\frac{q_{2}^{2}-\gamma^{2}}{1-\gamma^{2}}\right)^{1 / 2},
$$

where $\gamma=0.22$ is the intrinsic short-to-long axis ratio we use for the second galaxy. If the second galaxy has $q_{2} \leq 0.22$, it is assumed to be edge-on, resulting in $\cos \beta=1$.

Similarly, if we chose only those pairs which have at least one edge-on member, then we can assume $\cos i_{1} \approx 0$, and thus

$$
\cos \beta \approx \sin i_{2} \cos \phi \approx\left(\frac{1-q_{2}^{2}}{1-\gamma^{2}}\right)^{1 / 2}\left|\cos \left(\theta_{1}-\theta_{2}\right)\right| .
$$

Identifying the spiral galaxies that are very nearly edge-on or face-on, using photometric information alone, is necessarily approximate, given that spiral galaxies are not perfectly circular and do not all have the same intrinsic short-to-long axis ratio. Unterborn \& Ryden (2008) fitted the distribution of intrinsic thickness $\gamma$ with a Gaussian distribution; for luminous fracDeV $\leq 0.1$ galaxies in the $r$ band, they found a best fit of $\gamma=0.216 \pm 0.067$. Fitting the distribution of intrinsic disk ellipticity $\varepsilon$ with a lognormal distribution, Unterborn \& Ryden (2008) found $\ln \varepsilon=-2.56 \pm 0.91$, implying a median ellipticity $\varepsilon_{\text {med }}=0.077$.

For the purposes of this study, we assume that a galaxy with $q \geq 0.9$ is face-on, and a galaxy with $q \leq 0.3$ is edge-on. If spiral galaxies truly had the shape distribution given by Unterborn \& Ryden (2008) and quoted above, and were viewed from random angles, then the galaxies falling into our "face-on" class would have $\left\langle\cos ^{2} i_{1}\right\rangle=0.865$ and the galaxies in our "edge-on" class would have $\left\langle\cos ^{2} i_{1}\right\rangle=0.019$. Of our 747 isolated spiral galaxy pairs, $N_{f}=94$ pairs contain at least one face-on spiral and $N_{e}=171$ pairs contain at least one edge-on spiral. Since 14 pairs consist of a face-on spiral mated with an edge-on spiral, the total number of pairs with at least one face-on or edge-on spiral is $N_{\beta}=251$. 
In Figure 6, the black line shows the cumulative distribution function of $\cos \beta$ for the 171 galaxy pairs containing at least one edge-on spiral; for these pairs, $\cos \beta$ is computed using equation (9). The green line in Figure 6 shows the cumulative distribution function of $\cos \beta$ for the 94 pairs containing at least one face-on spiral; for these pairs, $\cos \beta$ is computed using equation (8) . Comparing the two distributions with a KS test, we find $P_{\mathrm{KS}}=0.823$. The two distributions are consistent with their being drawn from the same parent population of $\cos \beta$, which is what we expect in the absence of inclination-dependent selection effects. If the spiral galaxies in binary systems were randomly oriented with respect to each other, then the distribution of $\cos \beta$ would be uniform in the interval [0,1]. However, a KS test reveals that the distributions shown in Figure 6 are inconsistent, at a high level of statistical significance, with their being drawn from a uniform distribution of $\cos \beta$; for the pairs with an edge-on spiral, $P_{\mathrm{KS}}=0.021$, and for pairs with a face-on spiral, $P_{\mathrm{KS}}=0.004$. This is not necessarily an indication that the spiral galaxies are non-randomly oriented. The approximate values of $\cos \beta$ computed in equations (8) and (9) assume that spiral galaxies are perfect oblate spheroids, all with the same intrinsic axis ratio $\gamma$. These assumptions are known to be inexact.

For a more useful test of the randomness of the orientation of disks in a pair, we compare the distribution of the computed values of $\cos \beta$ for the isolated pairs with the distribution of $\cos i$ for the $N=10,458$ isolated spiral galaxies in our matched control sample. The values of $\cos i$, computed using equation (11), contain the same inexact approximations as the values of $\cos \beta$, computed using equations (8) and (91). In particular, the distribution of $\cos i$ for isolated spirals, given by the red line in Figure 6, should be directly comparable to the distribution of $\cos \beta$ for pairs including a face-on spiral, given by the green line in Figure 6; in both these cases, we are computing the cosine of the inclination of a spiral galaxy relative to the line of sight to that galaxy. However, having a face-on spiral within $R_{p}=1 \mathrm{Mpc}$ doesn't significantly affect a spiral galaxy's orientation relative to the line of sight; a KS test comparing the distribution of $\cos i$ for galaxies in the control sample and $\cos \beta$ for pairs with a face-on galaxy yields $P_{\mathrm{KS}}=0.431$.

Our statistical tests are consistent with the hypothesis that the two disks in an isolated binary spiral system are randomly oriented with respect to each other. However, since structure on scales larger than $\sim 1$ Mpc has an influence on tidal torques, we also investigate whether the number density of galaxies on a larger scale may influence the distribution of $\cos \beta$ for binaries. For each of our 171 pairs with a face-on member and 94 pairs with an edgeon member, we determine the number of neighboring galaxies that are within a projected distance of $R_{p}=5 \mathrm{Mpc}$ of either galaxy in the pair and with a radial velocity difference less than $\Delta v=300 \mathrm{~km} \mathrm{~s}^{-1}$ of either galaxy in the pair. To qualify as a neighboring galaxy, a galaxy must be in the Sloan spectroscopic sample, and its $r$ band absolute magnitude cannot 
be more than 1 magnitude dimmer than that of the lower-luminosity galaxy in the pair. The median number of neighboring galaxies, defined in this manner, was six. Consequently, we define pairs with six or fewer neighboring galaxies to be our "low-density" subsample, and pairs with more than six neighboring galaxies to be our "high-density" subsample.

The left panel of Figure 7 shows the distribution of $\cos \beta$ for pairs in the low-density subsample, while the right panel of Figure 7 shows the distribution for the high-density subsample. In each panel, the distribution of $\cos i$ for the isolated spiral galaxies in the matched control sample is repeated for reference (red line). If the simple picture of an isolated binary spiral system having parallel disks were correct, we might expect the pairs in the low-density subsample (left panel) to be show higher values of $\cos \beta$. In fact, we find that the distribution of $\cos \beta$ for both types of binary spiral systems (ones with edge-on disks and ones with face-on disks) is indistinguishable from the distribution of $\cos i$ for isolated spirals. The only marginally significant result we find, at the $\sim 90 \%$ confidence level, is that in high-density regions (right panel of Figure 7) binary spiral systems containing a face-on disk tend to have a distribution of $\cos \beta$ that is different from the distribution of $\cos i$ for isolated spirals: $P_{\mathrm{KS}}=0.088$. The difference is such that the partners of face-on disks are more likely to be seen edge-on than an isolated disk would be. In fact, of the 42 partners of face-on disks located in high-density regions, 6 have $q_{2}<0.22$. Of the isolated disks in the control sample, $4.9 \%$ have $q<0.22$; given a probability $P=0.049$ of an individual disk being edge-on, the expected probability of finding 6 or more edge-on galaxies in a sample of 42 would be $P=0.016$, using the standard binomial formula. Although, in high-density regions, the partners of face-on galaxies have a high probability of being edge-on, the partners of edge-on galaxies do not have a comparably high probability of being face-on.

\section{DISCUSSION}

In summary, our study of disk galaxies in the Sloan Digital Sky Survey reveals no strong evidence for a preferred alignment of the individual disk galaxies in a binary galaxy system. For 747 close pairs of galaxies, the distribution of $\phi$, the angle between the galaxies' projected major axes, is consistent with a uniform distribution between $0^{\circ}$ and $90^{\circ}$. This is consistent with disks that are randomly oriented with respect to each other.

Looking solely at $\phi$, however, doesn't use all the available information. Computing $\cos \beta$, where $\beta$ is the angle between the rotation axes of the disks, makes use of additional information - the apparent axis ratios of the two galaxies (equations 1 and 21). Taking advantage of the loss of tilt ambiguity when one disk is face-on or edge-on, we can compute the distribution of $\cos \beta$ for these special pairs. Looking at 171 close pairs with an edge- 
on galaxy and 94 close pairs containing a face-on galaxy reveals no statistically significant tendency for the two disk galaxies in a pair to be either parallel to each other $(\cos \beta \sim 1)$ or perpendicular to each other $(\cos \beta \sim 0)$.

We find one curious result that is consistent with correlated orientations. In relatively high-density neighborhoods (more than 6 bright neighboring galaxies with $R_{p} \leq 5 \mathrm{Mpc}$ ), face-on galaxies within a close pair have an unusually high probability of having a edge-on partner; although just $4.9 \%$ of all disk galaxies in our sample have $q<0.22,6$ out of 42 partners of face-on galaxies in high-density neighborhoods have $q<0.22$. The physical significance of this statistically significant result is unclear.

Obviously, the toy model for angular momentum acquisition, in which a pair of protogalaxies spin each other up and have antiparallel spin vectors and a tiny orbital angular momentum, is inconsistent with our results. Acquisition of spin angular momentum is supplied by torques from an array of protogalaxies and protogroups in the vicinity. Moreover, the initial angular momentum of a disk, acquired by tidal torques, can be modified by encounters and late infall. The continuing process of acquiring and modifying angular momentum appears, from our results, to produce binary spiral galaxies that have random relative orientations of their stellar disks.

We thank the referee for valuable advice. Funding for the SDSS and SDSS-II has been provided by the Alfred P. Sloan Foundation, the Participating Institutions, the National Science Foundation, the U.S. Department of Energy, the National Aeronautics and Space Administration, the Japanese Monbukagakusho, the Max Planck Society, and the Higher Education Funding Council for England. The SDSS website is http://www.sdss.org/. The SDSS is managed by the Astrophysical Research Consortium (ARC) for the Participating Institutions. The Participating Institutions are the American Museum of Natural History, Astrophysical Institute Potsdam, University of Basel, University of Cambridge, Case Western Reserve University, University of Chicago, Drexel University, Fermilab, the Institute for Advanced Study, the Japan Participation Group, Johns Hopkins University, the Joint Institute for Nuclear Astrophysics, the Kavli Institute for Particle Astrophysics and Cosmology, the Korean Scientist Group, the Chinese Academy of Sciences (LAMOST), Los Alamos National Laboratory, the Max-Planck-Institute for Astronomy (MPIA), the Max-PlanckInstitute for Astrophysics (MPA), New Mexico State University, The Ohio State University, University of Pittsburgh, University of Portsmouth, Princeton University, the United States Naval Observatory, and the University of Washington. 


\section{REFERENCES}

Adelman-McCarthy, K., et al. 2008, ApJS, 175, 297

Cervantes-Sodi, B., Hernandez, X., \& Park, C. 2010, MNRAS, 402, 1807

Chang, R., Shen, S., Hou, J., Shu, C., \& Shao, Z. 2006, MNRAS, 372, 199

de Vaucouleurs, G. 1948, Ann. d'Astrophys. 11, 247

Ellison, S. L., Patton, D. R., Simard, L., McConnachie, A. W. 2008, AJ, 135, 1877

Flin, P. 1993, AJ, 105, 473

Fukugita, M., Shimasaku, K., \& Ichikawa, T. 1995, PASP, 107, 945

Gott, J. R. III, Thuan, T. X. 1978, ApJ, 223, 426

Gunn, J. E., et al. 1998, AJ, 116, 3040

Han, O., Teyssier, R., \& Carollo, C. M. 2010, MNRAS, 405, 274

Helou, G. 1984, ApJ, 284, 471

Hoyle, F. 1949, in Burgers, J. M., \& van de Hulst, H. C., eds. Problems of Cosmical Aerodynamics. Central Air Documents Office, Dayton, OH, p. 195

Jiang, C. Y., Jing, Y. P., Faltenbacher, A., Lin, W. P., \& Li, C. 2008, ApJ, 675, 1095

Karachentsev, I. D. 1972, SoSAO, 7, 1

Lacey, C., \& Cole, S. 1993, MNRAS, 262, 627

Oosterloo, T. 1993, A\&A, 272, 389

Padilla, N. D., \& Strauss, M. A. 2008, MNRAS, 288, 1321

Patton, D. R., Ellison, S. L., Simard, L., McConnachie, A. W., \& Mendel, J. T. 2011, MNRAS, 412, 591

Paz, D. J., Sgró, M. A., Merchán, M., \& Padilla, N. 2011, MNRAS, 414, 2029

Paz, D. J., Stasyszyn, F., \& Padilla, N. 2008 MNRAS, 389, 1127

Peebles, P. J. E. 1969, ApJ, 155, 393

Perez, J., Tissera, P., \& Blaizot, J. 2009, MNRAS, 397, 748 
Pestaña, L. G., \& Cabrera, J. 2004, MNRAS, 353, 1197

Schneider, M. D., Frenk, C. S., \& Cole, S. 2011, JCAP, submitted (arXiv:1111.5616)

Shao, Z., Xiao, Q., Shen, S., Mo, H. J., Xia, X., \& Deng, Z. 2007, ApJ, 659, 1159

Sharp, N., Lin, D. N. C., \& White, S. D. M. 1979, MNRAS, 187, 287

Slosar, A., et al. 2009, MNRAS, 305, 357

Smith, J. A., et al. 2002, AJ, 123, 2121

Sofue, Y. 1992, PASJ, 44, L1

Strateva, I., et al. 2001, ApJ, 122, 1861

Unterborn, C. T., \& Ryden, B. S. 2008, ApJ, 687, 976

Vincent, R. A., \& Ryden, B. S. 2005, ApJ, 623, 137 


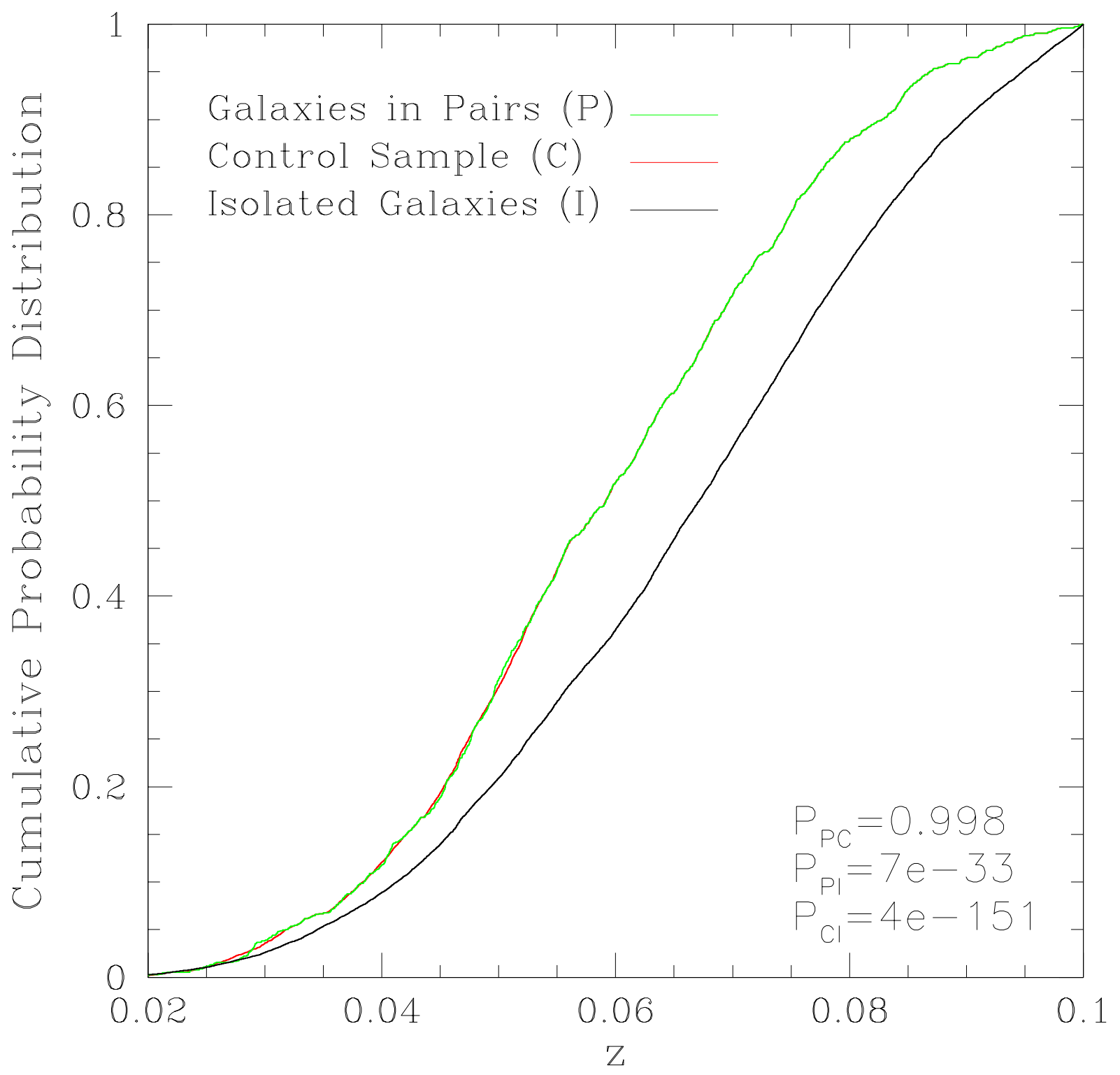

Fig. 1.- The cumulative distribution of redshift $z$ for the 1494 disk galaxies in pairs (green), for the matched control sample of $7 \times 1494=10,458$ isolated disk galaxies (red), and for the complete sample of 16,814 isolated disks (black). 

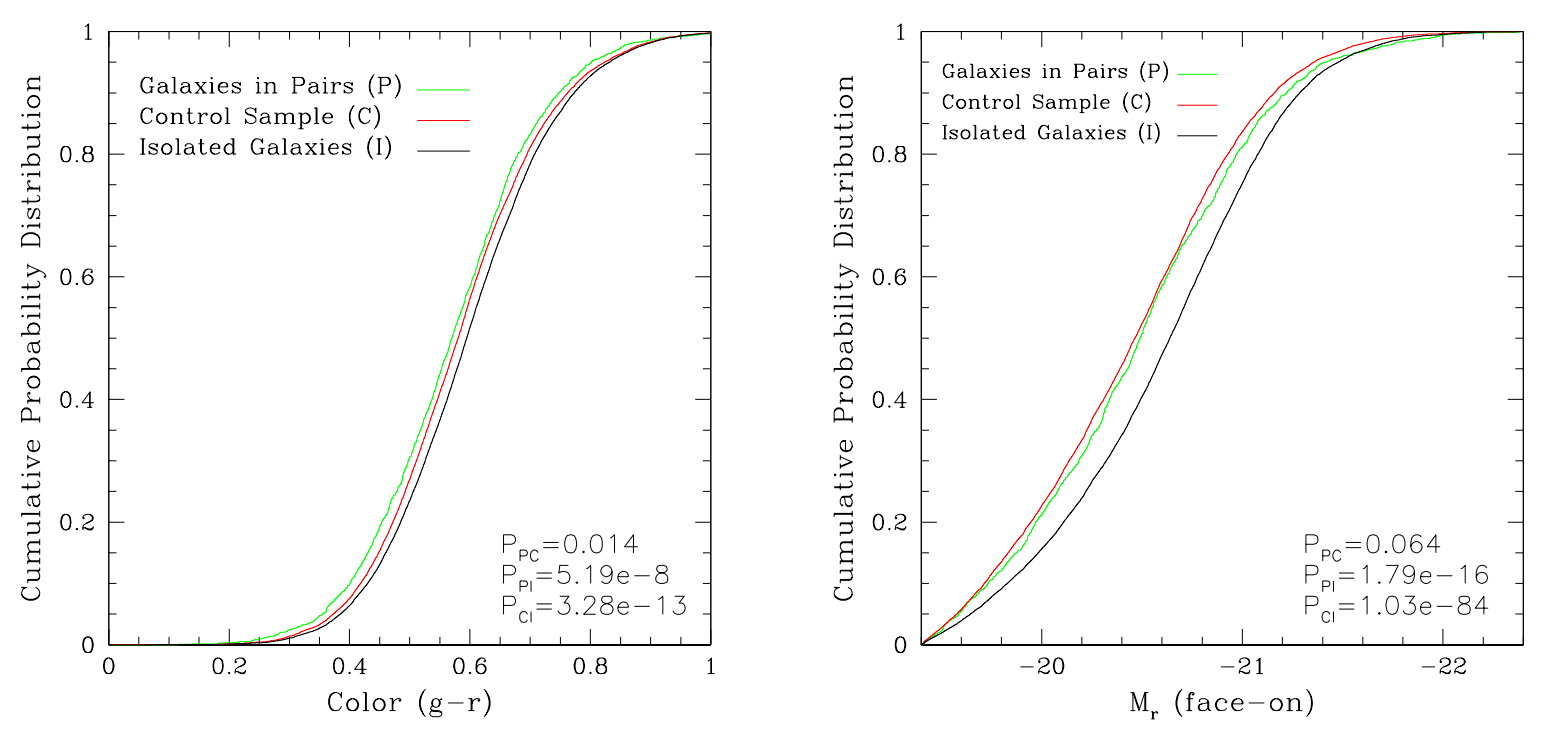

Fig. 2.- Left panel: The cumulative distribution of $g-r$ color for the 1494 disk galaxies in isolated pairs (green), for the matched control sample of 10,458 isolated disk galaxies (red), and for the complete sample of 16,814 isolated disks (black). Right panel: The cumulative distribution of $M_{r}$, corrected to face-on using the presciption of Unterborn \& Ryden (2008) for the disk galaxies in isolated pairs (green), for the matched control sample of isolated disks (red), and for all isolated disks (black). 


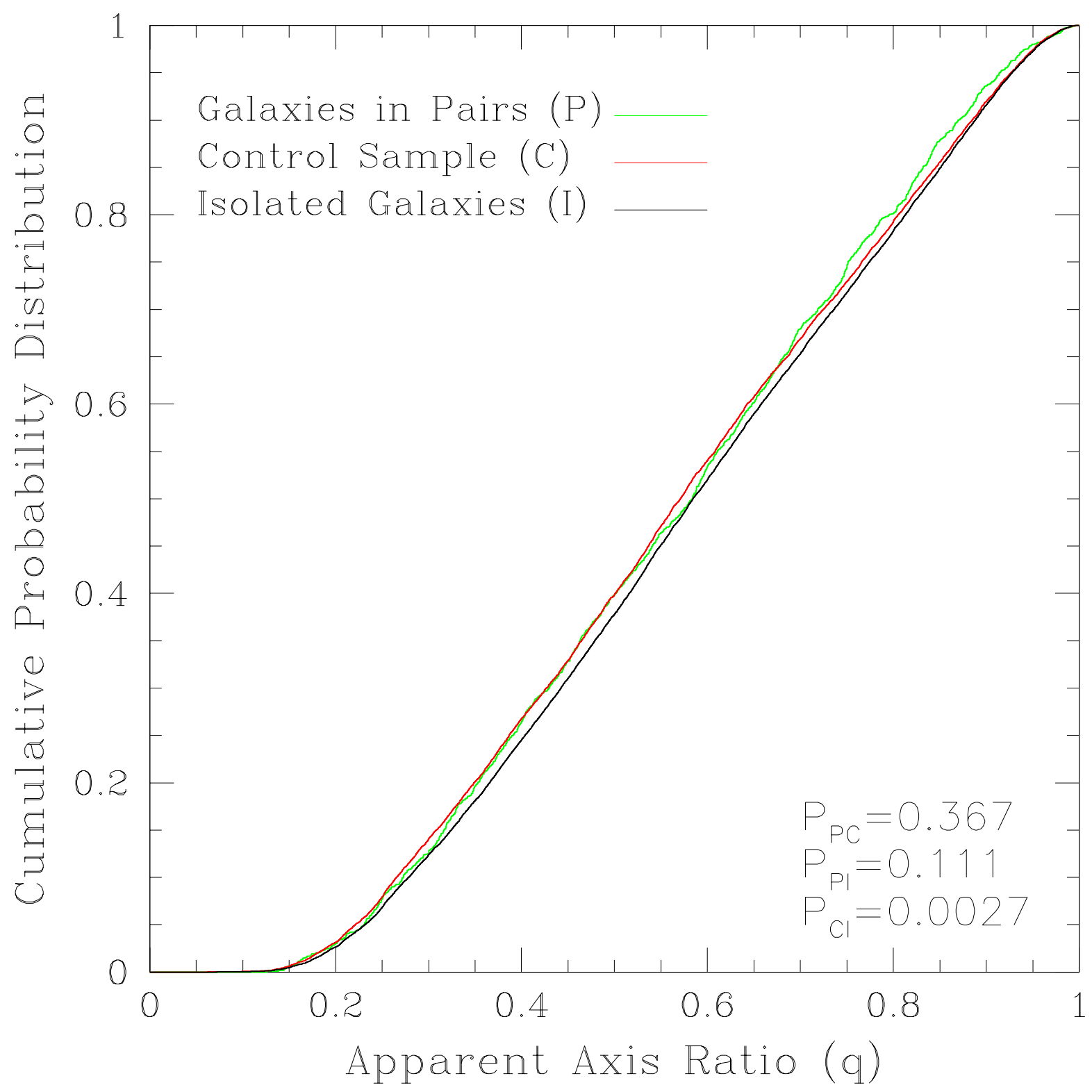

Fig. 3.- The cumulative distribution for the apparent axis ratios $q$ of the 1494 disk galaxies in isolated pairs (green), for the matched control sample of 10,458 isolated disks (red), and for the complete sample of 16,814 isolated disks (black). 


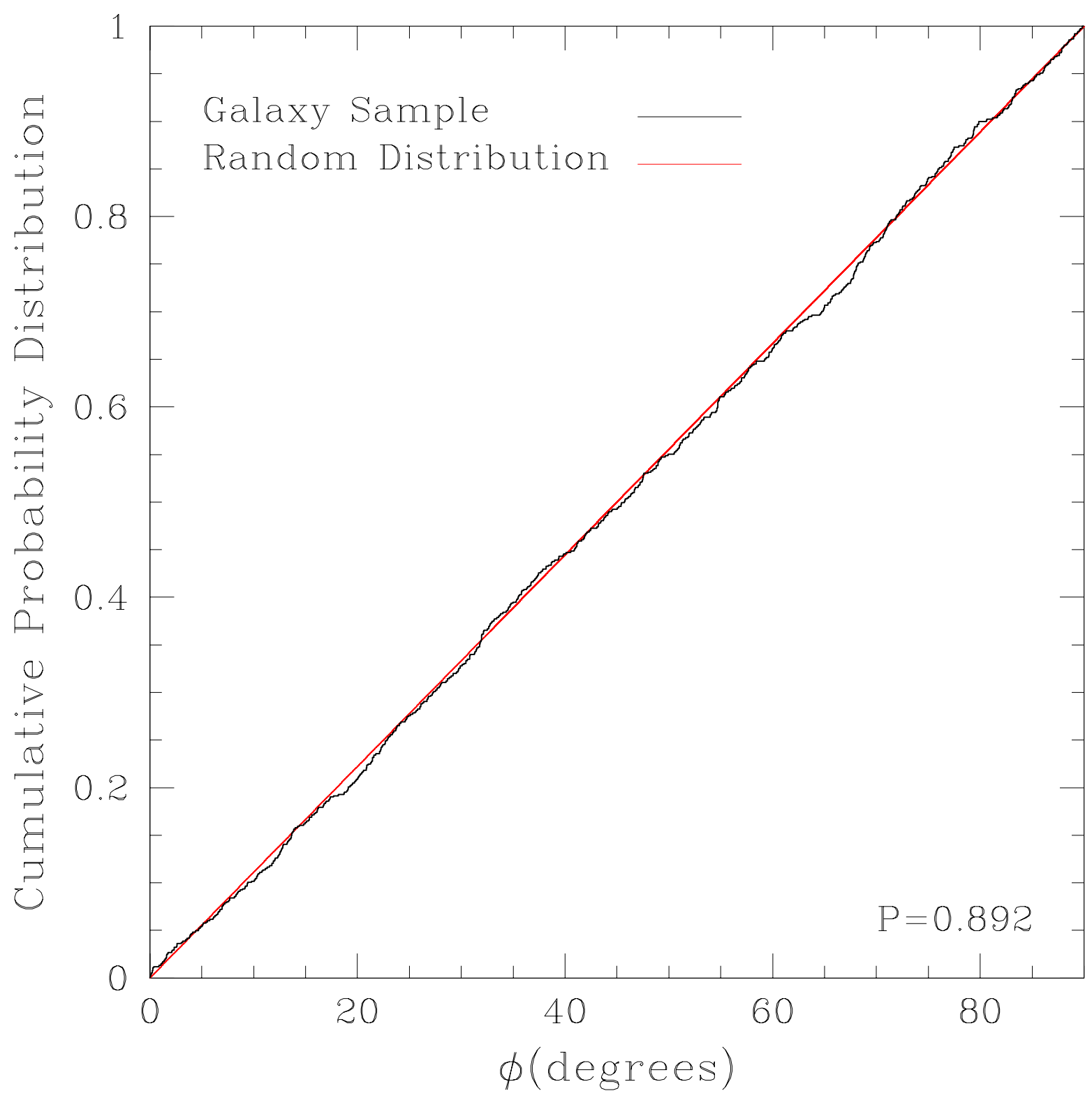

Fig. 4. - The cumulative distribution of the angle $\phi$ between the major axes of the galaxy images in the 747 isolated pairs of disk galaxies in our main sample (black); for comparison, a uniform distribution of $\phi$ is shown as the red line. 


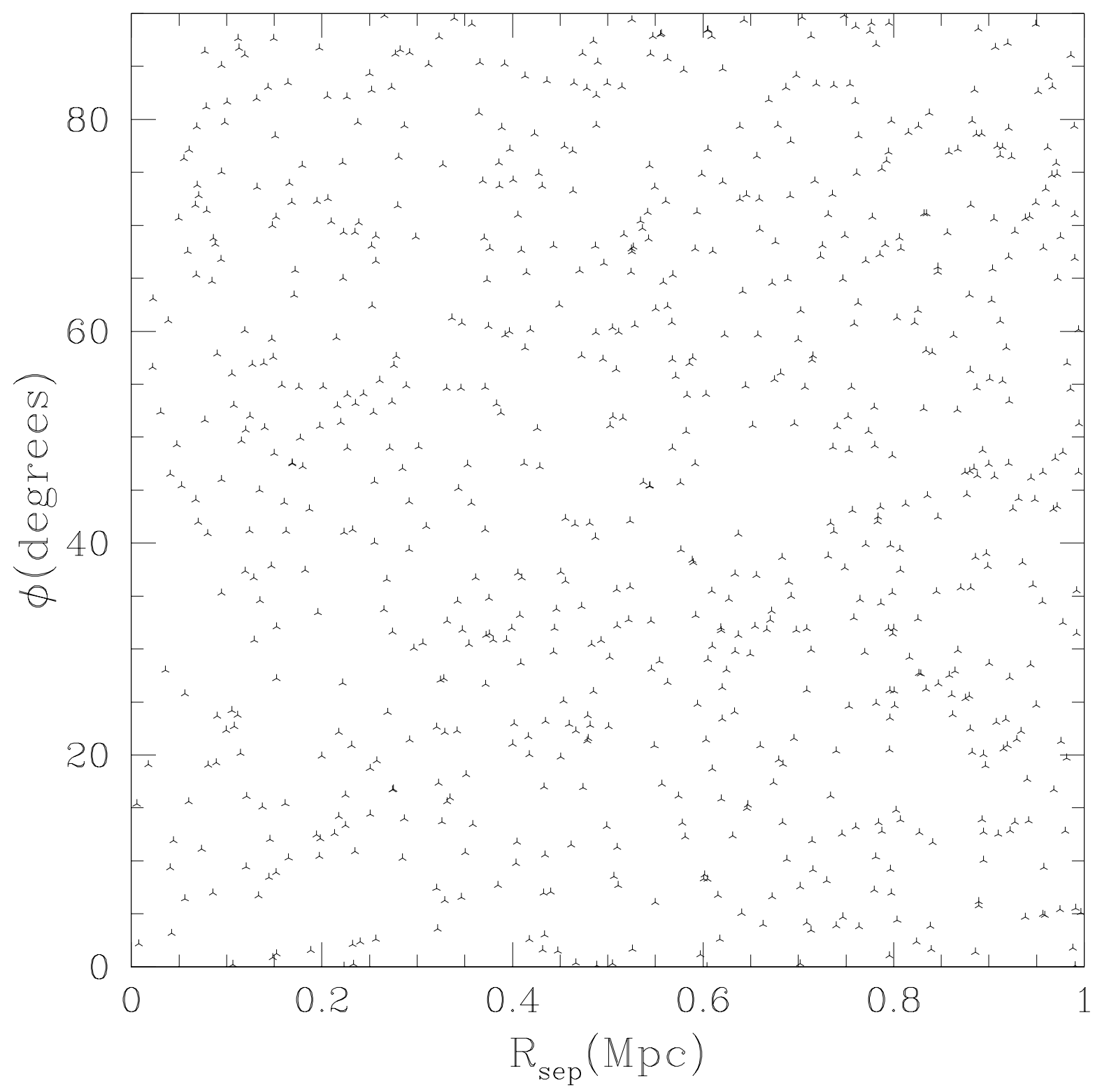

Fig. 5.- The angle $\phi$ between the major axes of the galaxies images in the 747 isolated pairs, plotted as a function of the projected separation $R_{p}$ between the galaxy centers. 


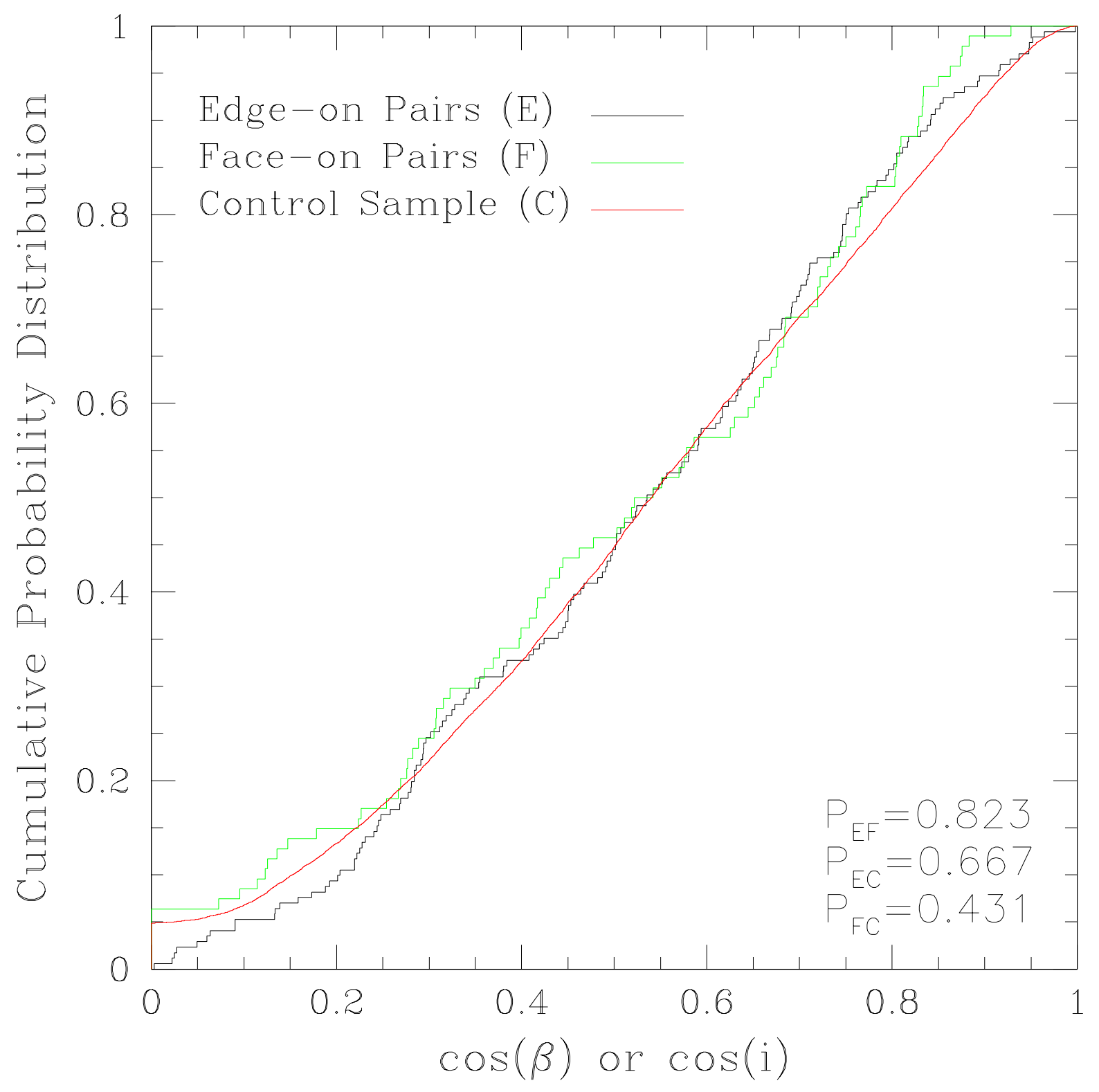

Fig. 6.- The green line shows the cumulative distribution of $\cos \beta$, estimated from equation (8), for the $N_{F}=94$ isolated pairs in which at least one galaxy is face-on. The black line shows the distribution of $\cos \beta$, estimated from equation (99), for the $N_{E}=171$ pairs in which at least one galaxy is edge-on. A galaxy is judged to be edge-on if $q \leq 0.3$ and face-on if $q \geq 0.9$. For comparison, the red line shows the distribution of $\cos i$, estimated from equation (1), for the matched control sample of isolated disks. 

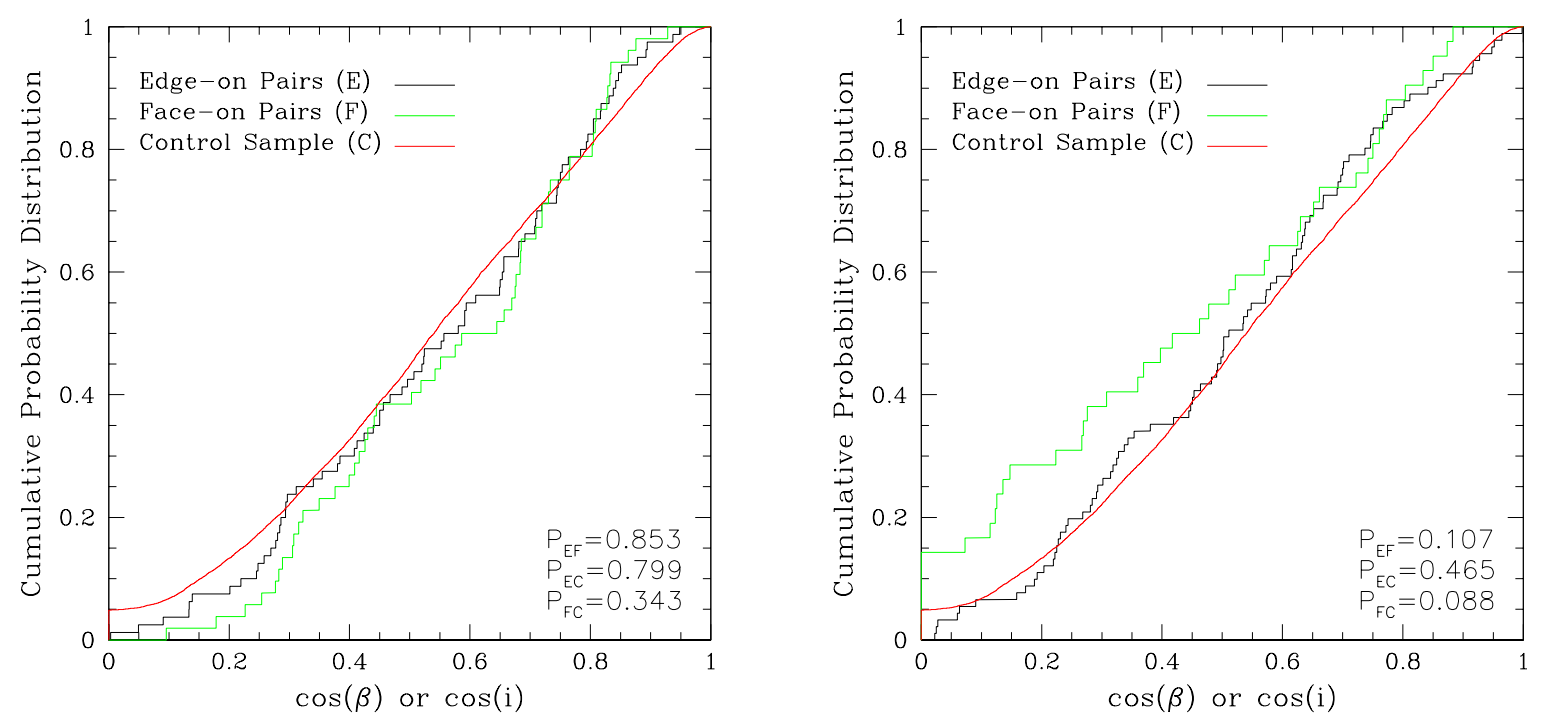

Fig. 7.- The cumulative distribution of $\cos \beta$ for pairs in the low-density subsample (left panel) and high-density subsample (right panel). Within each panel, the black line indicates pairs with at least one edge-on disk and the green line indicates pairs with at least one face-on disk. For comparison, the red line shows the distribution of $\cos i$ for the matched control sample of isolated disks. The values of $\cos \beta$ and $\cos i$ are estimated as described in the caption of Figure 6 . 